\title{
Geschätzte Leser/innen!
}

Das Titelbild dieses Heftes zeigt - für unsere Stammleser/innen wohl verwunderlich - ein „schlichtes“ Landschaftsmotiv. Hierbei handelt es sich um den Ötscher, einen 1893 m hohen Berg in den Nördlichen Kalkalpen Niederösterreichs. Dort stellt er mit seiner Form, herausragenden Höhe und weiten Sichtbarkeit eine Singularität dar, die ihm schon seit Jahrhunderten eine besondere Wertschätzung durch die ortsansässige Bevölkerung, aber auch schon früh Aufmerksamkeit von Seiten der Wissenschaft beschert hat. 2015 hat die Niederösterreichische Landesausstellung „ÖTSCHER.REICH. Die Alpen und wir" dem Berg wieder zu einiger Publicity verholfen, wobei der Bergname auch symbolhaft für eine neue regionale Identität als Ausgangspunkt und Katalysator einer nachhaltigen Regionalentwicklung aufgeladen wurde. Was das alles mit dem Heft zu tun hat? Nun, der Zufall wollte es, dass zwei Beiträge den Weg in dieses Heft gefunden haben, worin der Ötscher mitsamt dem Aspekt der Regionalentwicklung in seinem weiteren Umfeld zumindest implizit zur Sprache kommt. Es sind dies zum einen der fachwissenschaftliche Artikel von Janis Dinter, Vanessa Färber, Lukas Kindl, Anja Speyer, Juliane Weickert, Robert Steiger und Bruno Abegg, worin in der Diskussion um touristische Wettbewerbsfähigkeit ländlicher Gebiete einer der Untersuchungsräume das „Ötscherland“ beinhaltet. Und zum anderen bieten wir Ihnen im Serviceteil wieder eine Interpretation eines Kartenausschnittes jener Freytag \& Berndt-Wanderkarte, auf der auch der Ötscher zu sehen ist.

Wie schon angedeutet, hat dieses Heft keinen thematischen Schwerpunkt, sondern bietet Ihnen einen bunten „Strauß“ an unterschiedlichen Beiträgen. In der Rubrik Fachdidaktik fokussiert Birte Schröder auf machtsensible Konzepte interkulturellen Lernens und macht mit ihrem Vorschlag GW-Didaktik zum in der Fachwissenschaft hochaktuellen Diskurs des Postkolonialismus anschlussfähig. In der Rubrik Unterrichtspraxis finden Sie zwei Beiträge, die sich zentralen Unterrichtsprinzipien des GW-Unterrichts widmen: Arnela Alic, Alexander Hoffelner, Ernst Königshofer und Sabrina Gölles nehmen die politische Bildung über das Thema Verkehr in der Stadt in den Blick, während Martin Dür, Christian Reiner und Christian Vielhaber sozioökonomische Bildung über prekäre Arbeitswelten und Konsumerwartungen thematisieren. Dieser Beitrag besitzt einen inhaltlichen Zusammenhang mit dem im Serviceteil enthaltenen Tagungsbericht von Herbert Pichler und Viola Kessel. Im Frühjahr 2016 fand in Schlierbach (Oberösterreich) eine Tagung zur GW-Fachdidaktik statt, an der Vertreter/innen aller österreichischen Universitäts- und Hochschulstandorte, an denen ein GW-Studium angeboten wird, teilnahmen. Das Tagungsthema lautete „(Schöne) Neue Arbeitswelten - zwischen New Economy und Prekarisierung“. Wegen des großen Erfolgs dieser Veranstaltung wird es auch 2017 wieder eine solche Tagung geben, die von einem kleinen Team bereits eifrig vorbereitet wird.

Eine weitere erfreuliche Nachricht für die GW-Didaktik können wir weitergeben: Im Frühsommer 2016 konstituierte sich innerhalb der Österreichischen Geographischen Gesellschaft (ÖGG) eine „Fachgruppe für geographische und sozioökonomische Bildung", die von Christian Fridrich, Maria Hofmann-Schneller und Alfons Koller geleitet wird. Auch an ihr sind Vertreter/innen aller österreichischen Standorte der GW-Ausbildung beteiligt. Damit gibt es nun in der ÖGG - neben Gruppen für Kartographie, Angewandte Geographie sowie Geomorphologie und Umweltwandel - wieder eine vierte Fachgruppe, die die Anliegen der GW-Fachdidaktik vertritt und zum Ausdruck bringt, dass auch die ÖGG der Fachdidaktik einen hohen Stellenwert beimisst. Sie sind herzlich eingeladen, dieser Gruppe als Mitglied beizutreten bzw. sich darin im Sinne unseres gemeinsamen Anliegens zu engagieren. Nähere Informationen werden in Kürze über die Homepage der ÖGG (http://www. geoaustria.ac.at/) aufrufbar sein.

Ganz und gar nicht erfreulich für unser Fach waren hingegen die Äußerungen von Andreas Treichl, Chef der Erste Group, der anlässlich der Eröffnung eines Finanzbildungszentrums (Erste Financial Life Park) am 28.10.2016 in Wien das Fach und seine Lehrer/innen scharf kritisierte. Da es sich dabei jedoch in keiner Weise um eine qualifizierte, konstruktive Kritik, sondern um eine ungerechtfertigte und in der Wortwahl disqualifizierende Polemik handelte, bringen wir im Kontrapunkt eine aktuelle, dringend notwendige Entgegnung. 43 Personen aus der GW-Aus- und Fortbildung sowie - Unterrichtspraxis haben diesen Text unterzeichnet.

Viel Freude beim Lesen, einen erholsamen Jahreswechsel und ein erfolgreiches, erfülltes und durch die Lektüre von GW-Unterricht bereichertes Jahr 2017 wünschen Ihnen herzlichst 